\title{
Intrinsic Notch Effect Leads to Breakdown of Griffith Criterion in Graphene
}

\author{
Feng Liu, * Qiheng Tang, and Tzu-Chiang Wang*
}

\begin{abstract}
Due to lack of the third dimension in 3D bulk materials, the crack tip in graphene locates on several atoms implying that its fracture behavior can be closely associated with its lattice structure, i.e., the bond length and angle. As the bond length reflects the discrete nature of the atomic structure, theoretical discussion is focused on the concomitant size effect at the nanoscale with few or no reports about the influence of the bond angle. Through the comparisons between theoretical calculations and experimental data, here it is first demonstrated that the bond angle is essential for understanding the fracture behavior in graphene, serving as an intrinsic notch reducing the stress singularity near the crack tip (the intrinsic notch effect), leading to the breakdown of the Griffith criterion in graphene. The work provides a framework for the studying of the brittle fracture in 2D materials, which gives rise to the more reliable device design based on 2D materials. More importantly, the significance of the intrinsic notch effect is profound and far-reaching, paving the way to a more comprehensive and deep understanding of the mechanical properties in nano as well as nanostructured materials.
\end{abstract}

\section{Introduction}

Graphene, a one-atom thick sp2-bonded carbon sheet, exhibits remarkable electronic, ${ }^{[1,2]}$ thermal, ${ }^{[3]}$ and optical ${ }^{[4]}$ properties. In its perfect form, the mechanical properties of graphene are equally outstanding, with a Young's modulus of $1 \mathrm{TPa}$ and mechanical strength above $90 \mathrm{GPa}$, exceeding that of steel. ${ }^{[5,6]}$ Combining the advantages of its superior physical and mechanical attributes, prefect graphene could be a good candidate in applications such as flexible

Dr. F. Liu, Prof. Q. Tang, Prof. T.-C. Wang

State Key Laboratory of Nonlinear Mechanics Institute of Mechanics

Chinese Academy of Sciences

Beijing 100190, China

E-mail: liufeng@imech.ac.cn; tcwang@imech.ac.cn

Prof. Q. Tang, Prof. T.-C. Wang

School of Engineering Science

University of Chinese Academy of Sciences

Beijing 100049, China

DOI: $10.1002 / \mathrm{smll} .201700028$ electronics, ${ }^{[7-9]}$ electromechanical devices, ${ }^{[10]}$ and composite reinforcement, ${ }^{[11]}$ and also an ideal platform for strain engineering, ${ }^{[12]}$ for example, the wrinkling in graphene could be used to manipulate the size of the electronic band gap arousing intense academic interests. ${ }^{[13-18]}$ However, as the second law of thermodynamics dictates, the presence of disorders is inevitable on large-scale graphene samples. Indeed, up to date, the existing methods of large-scale production of graphene are known to introduce a certain degree of defects in samples. Compared with other defects, crack-like ones may cause the dramatic degradation of the strength, which is detrimental to the practical applications of graphene. Therefore, rather than its intrinsic strength, the engineering relevant one turns out to be determined by its fracture toughness, i.e., the ability of a material containing a crack to resist fracture.

Despite great theoretical and experimental efforts have been made to investigate the fracture toughness in graphene over the last two decades, ${ }^{[19-31]}$ there are still controversies, especially, the critical crack size below which the Griffith criterion of brittle fracture breaks down. A recent investigation shows that the critical crack size for the Griffith criterion is $1 \mathrm{~nm}$ with cracks no more than $3 \mathrm{~nm}$ in length considered 
in this study, ${ }^{[26]}$ in contrast to $10 \mathrm{~nm}$ suggested based on large-scale molecular dynamics (MD) simulations, ${ }^{[31]}$ where the longest crack length is over $30 \mathrm{~nm}$. It seems that on different length scales, the critical size for the Griffith criterion changes, which raises a question whether the Griffith criterion is still reasonable in graphene.

Consisting of a single layer of carbon atoms, graphene is a 2D material, in which the crack tip locates on several atoms due to lack of the third dimension in 3D bulk materials, and therefore the lattice structure, i.e., the bond length and angle, could be of crucial importance to its fracture behavior. As the bond length reflects the discrete nature of the atomic structure, quantized fracture mechanics, ${ }^{[32]}$ the finite difference version of the Griffith criterion, has recently been developed and used to explain the nanoscale fracture behaviors in graphene ${ }^{[33,34]}$ and also in carbon nanotube. ${ }^{[35]}$ Nevertheless, the influence from the bond angle has not been scratched yet. Through the comparisons between theoretical calculations and experimental data, here we first demonstrate that the bond angle can be essential for understanding the fracture behavior in graphene, serving as an intrinsic notch reducing the stress singularity near the crack tip (the intrinsic notch effect), leading to the breakdown of the Griffith criterion in graphene. Although the notch effect has been discussed long ago in fatigue and fracture, as well as its key role in the application of structural materials recognized, ${ }^{[36]}$ it is usually considered as an external geometrical effect. As the notch effect becomes intrinsic in graphene, its influence to the fracture behavior in this system needs to be explored.

\section{Results}

\subsection{Intrinsic Notch in Graphene}

The Griffith criterion and the stress intensity factor theory are now the most fundamental theories for characterizing the fracture behavior of brittle materials and have been heavily applied in engineering design. Although the viewpoints for those two criteria are quite different (energy balance viewpoint is provided by the Griffith criterion, while the stress singularity at the crack tip is emphasized in the stress intensity factor theory), the same dependence for the critical fracture stress $\sigma_{f}$ and crack length $a$ could be obtained, i.e., $\sigma_{f} \sim a^{-1 / 2}$. However, the discrepancy emerges, if the notch effect is taken into account. Specifically, for a notch with an infinite acuity, the stress singularity follows $\sigma_{i j} \sim r^{-\alpha}$, where $\alpha$ is the singular exponent and deviates from 0.5 with a nonzero notch angle. ${ }^{[36]}$ Since the fracture criterion in the stress intensity factor theory is $K_{i}=K_{i ; c}$, where $i=\mathrm{I}$, II, III and $K_{i, \mathrm{c}}$ is the fracture toughness of Mode $i$, the critical fracture stress $\sigma_{f}$ scales as $a^{-\alpha}$ according to the dimensional analysis, if the internal crack length $a$ is considered as the only related characteristic length scale. It is qualitatively different from $\sigma_{f} \sim a^{-1 / 2}$ predicted by the Griffith criterion, ${ }^{[37]}$ where the notch effect is not considered, in other words, at least one of these fracture criteria fails in that case. Fortunately, a notch with an infinite acuity seems to exist only in mathematics, besides much attentions have been paid on the blunted notch with a nonzero radius but zero angle alleviating this discrepancy to a certain extent.

This is true for 3D bulk materials, since a notch with an infinite acuity is hard to achieve via the external processing. However, the situation changes in graphene due to its honeycomb lattice structure formed by covalent carbon-carbon bonds (see Figure 1a left). As the bond force is always along the bond direction, the covalent bond serves as a free boundary and thus the bond angle is equivalent to a notch with an infinite acuity (see Figure 1a right). Surprisingly, without any external processing the honeycomb lattice structure of graphene naturally introduces intrinsic notches into this system. More interesting, any form of the crack tip is composed by these intrinsic notches (see Figure 1b), and therefore the corresponding intrinsic notch effect in graphene is robust and should be carefully examined. Note that the so-called "intrinsic notch" is used to stress the inartificial and robust nature of these bond angles.

The stress distribution of a notch with an infinite acuity in an infinite body (see the inset in Figure 1c) has already been a

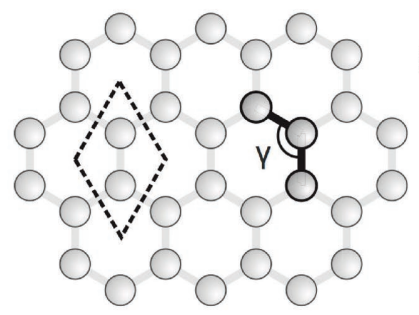

C

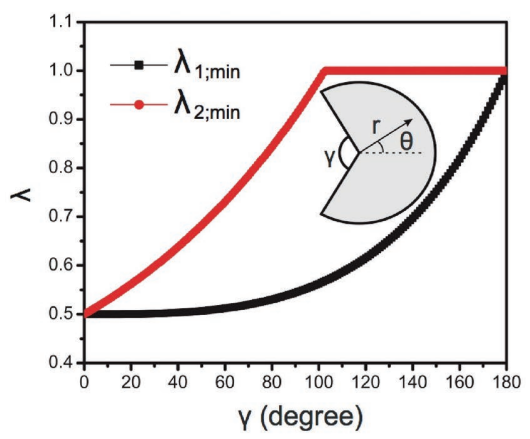

b

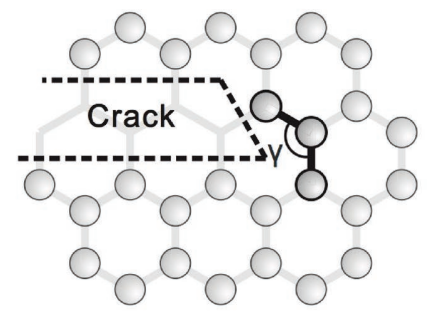

d

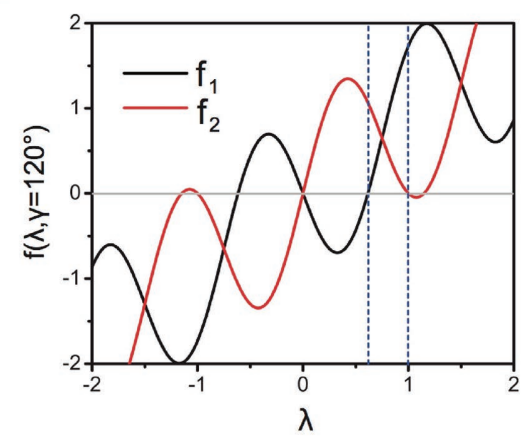

Figure 1. The origin of intrinsic notches and the corresponding stress singularity. a) The honeycomb lattice structure of graphene (left) where the unit cell and the intrinsic notch are marked. The schematic diagram of the intrinsic notch effect (right): as the bond force is always along the bond direction, the bond serves as a free boundary and the bond angle is equivalent to a notch with an infinite acuity. b) The configuration of a crack tip, which is composed by intrinsic notches. c) The evolution of $\lambda$ with respect to the notch angle $\gamma$, note that \pm in Equation (1) gives two sets of solutions, and the minimum solutions for $\lambda>0$ labeled $\lambda_{1 ; \min }$ (for - ) and $\lambda_{2 ; \min }$ (for + ) are shown. d) The function $f(\lambda, \gamma=2 \pi / 3)= \pm \sin (\lambda(2 \pi-\gamma))-\lambda \sin (2 \pi-\gamma)$ versus $\lambda$. Two function values are labeled with $f_{1}$ (for - ) and $f_{2}$ (for + ), respectively. Note that $f=0$ is equivalent to Equation (1). 
studied, ${ }^{[36]}$ and the stress singularity is found to be dependent on the notch angle. The eigen equation which determines the stress singularity is given as (the details of the derivation could be found in the Supporting Information):

$\sin (\lambda(2 \pi-\gamma))= \pm \lambda \sin (2 \pi-\gamma)$

where $\gamma$ is the notch angle and $\lambda$ is related to the singular exponent $\alpha(\alpha=1-\lambda)$, which can be seen from the expression of the corresponding stress field $\sigma_{i j}=r^{-(1-\lambda)} f_{i j}(\lambda, \theta)$, where $(\theta, r)$ is the polar coordinate (see the inset in Figure 1c), and $f_{i j}(\lambda, \theta)$ are functions of $\lambda$ and $\theta$. Considering the strain energy convergence condition $\lambda$ should be greater than 0 , and the minimum solutions of Equation (1) for $\lambda>0$ are shown in Figure $1 \mathrm{c}$. It indicates that $\alpha=1-\lambda$ equals 0.5 only if the notch angle equals $0^{\circ}$, and it decreases with the increasing notch angle. Since the intrinsic notch angle equals $120^{\circ}$ in graphene, the corresponding solutions for Equation (1) are shown in Figure 1d via the graphic method. Note that when the notch angle is less than $103^{\circ}$, there are two solutions within $0<\lambda<1$ for Equation (1) suggesting different stress singularities for two fracture modes (see Figure 1c), i.e., Mode I (opening mode) and Mode II (sliding mode). As a consequence, different loading modes may cause different stress singularities, and for the mixed-mode loading (Mode I + Mode II) the fracture behavior could be different on different scales. Fortunately, for the $120^{\circ}$ notch, there is only one solution for Equation (1), i.e., $\lambda=0.6157$ for $0<\lambda<1$ (see Figure $1 d$ ), corresponding to Mode I, which implies the robust fracture behavior in graphene.

\subsection{Breakdown of the Griffith Criterion}

As mentioned above, when the notch effect is taken into account, the discrepancy emerges between the Griffith criterion and the stress intensity factor theory, so the question is which one prevails in graphene. In this article, MD simulations are used to gain insights into the fracture criterion in graphene, and the details of our simulations can be found in the Experimental section. Figure 2a shows a schematic representation of the central crack model in our simulations, where the tensile loading direction is along the armchair direction. Figure $2 \mathrm{~b}$ shows the snapshot of the graphene sheet involving a $19.23 \mathrm{~nm}$ central crack subject to $1 \%$ tensile strain (the overall view can be found in the inset of Figure $2 b$ ), where the color reflects the distribution of the virial stress $\sigma_{y y}$. Consistent with theoretical supposition, there is a stress concentration at the crack tip, and along the horizontal direction the stress distribution is plotted in Figure 2c. The red solid line in Figure $2 \mathrm{c}$ is fitted to the stress distribution ahead of the crack tip obtained in MD simulations by adjusting the coefficient $\mathrm{B}$ and the singular exponent $\alpha$ in $\sigma_{y y}=\mathrm{Br}^{-\alpha}$. Note that the singular exponent $\alpha=0.393$ obtained by fitting (see Figure 2c) coincides with the one for the $120^{\circ}$ notch $(\alpha=0.3843$ predicted by Equation (1)) demonstrating the existence of the intrinsic notch effect. With this intrinsic notch effect, the stress concentration could be reduced, and in this sense graphene is tougher than expected. The comparison between our MD results and the solution of the stress field near a sharp crack tip can be found in Figure S2 (Supporting Information). a

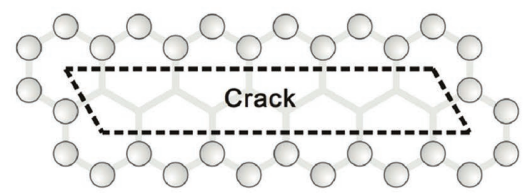

b

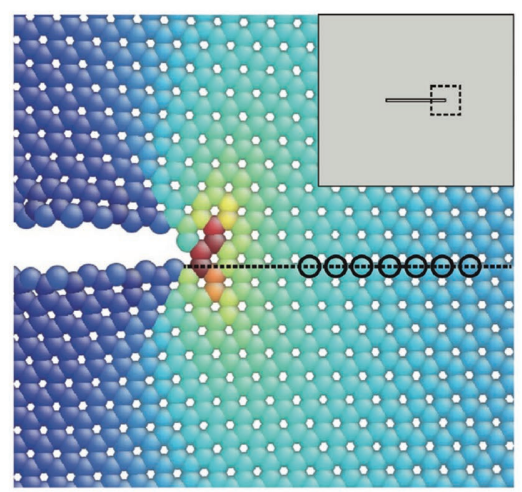

C

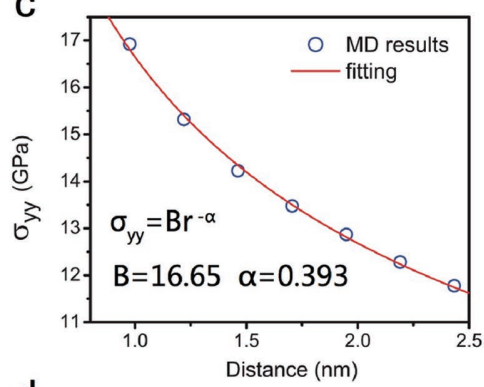

d

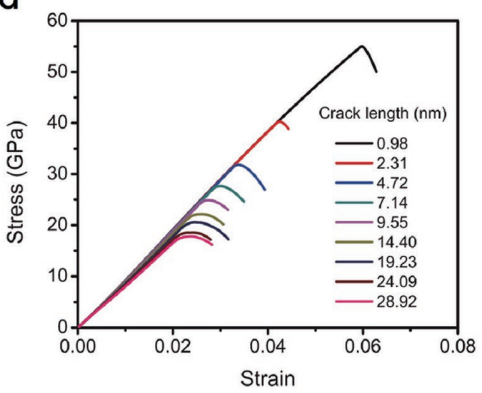

e

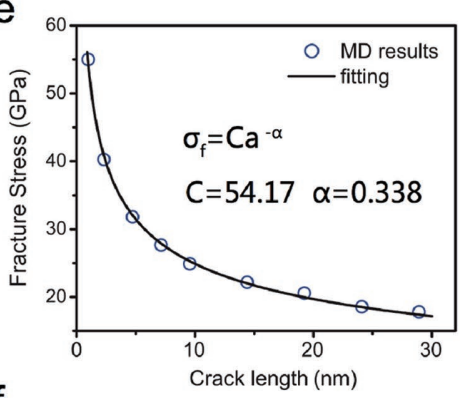

f

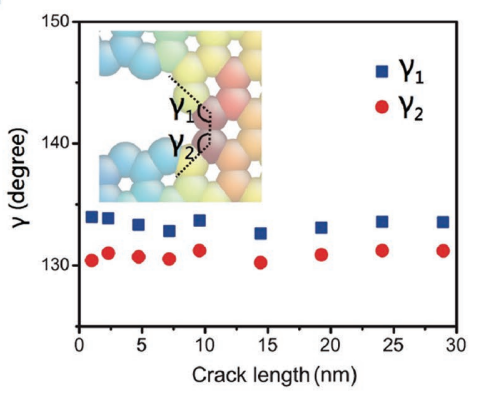

Figure 2. The fracture behavior in graphene with the tensile loading direction along the armchair direction. a) The schematic diagram of the configuration of the central crack in graphene, note that the crack is along the zigzag direction while the loading direction is along the armchair direction. b) The snapshot of the graphene sample involving a $19.23 \mathrm{~nm}$ central crack subject to $1 \%$ tensile strain, where the color reflects the distribution of the virial stress $\sigma_{y y}$. Note that the overall view is given in the inset, and the snapshot region is marked with the dashed box. c) The stress distribution along the horizontal direction and the corresponding atoms are marked with black circles in (b). d) The tensile stress-strain curves for graphene samples with 1-30 nm length central cracks are shown. e) The evolution of the critical fracture stress with respect to the crack length. f) The bond angles at the crack tip (at about $0.3 \%$ strain preceding the onset of the fracture) versus the crack length. Two bond angles are marked in the inset. 
As the stress singularity is well captured, according to the stress intensity factor theory the critical fracture stress $\sigma_{f}$ scales as $a^{-\alpha}$, and the Griffith criterion should break down.

To corroborate this, tensile MD simulations are performed for graphene samples with 1-30 nm length central cracks, and the tensile stress-strain curves are shown in Figure 2d. In Figure 2e, the evolution of the critical fracture stress with respect to the crack length is shown, and the MD results are fitted by $\sigma_{f}=C a^{-\alpha}$, where $\alpha=0.338<0.5$ indicating the breakdown of the Griffith criterion in graphene. However, $\alpha=0.338<0.393$ also shows a considerable derivation from the stress intensity factor theory. To understand this derivation, the two bond angles at the crack tip are monitored (marked with $\gamma_{1}$ and $\gamma_{2}$ in the inset of Figure 2f). As shown in Figure $2 \mathrm{f}, \gamma_{1}$ and $\gamma_{2}$ at about $0.3 \%$ strain preceding the onset of the fracture are independent of the crack length and approximately equal to $133^{\circ}$ and $131^{\circ}$, respectively. In fact, before the onset of the fracture only these two bond angles at the crack tip are greatly changed during the tensile test, other bond angles at the front of the crack tip are nearly unchanged and roughly $120^{\circ}$. These observations suggest that due to the brittle nature of graphene, ${ }^{[38]}$ its fracture process region is confined to a small area, and it is supposed that when approaching the onset of the fracture, the bond angle of $132^{\circ}$ at the crack tip (the average of $\gamma_{1}$ and $\gamma_{2}$ ) determines the evolution of the critical fracture stress with respect to the crack length, while the bond angles away from the crack tip (roughly $120^{\circ}$ ) determine the pseudosingularity of the stress field. Indeed, with a notch angle of $132^{\circ}$, the singular exponent $\alpha$ equals 0.34 (predicted by Equation (1)), which is in great agreement with $\alpha=0.338$ obtained in our MD simulations. Since only the tensile test along the armchair direction is performed in graphene, the existence of the intrinsic notch effect and also the breakdown of the Griffith criterion need to be further confirmed by considering the influence of the lattice orientation, which will be discussed in the next section.

\subsection{Influence of the Lattice Orientation}

For the convenience of discussion, the angle $\varphi$ is used to define the lattice orientation (see Figure 3a). The tensile tests of the perfect graphene for $\varphi=0^{\circ}, 7.6^{\circ}, 15^{\circ}, 22.3^{\circ}$, and $30^{\circ}$ are performed, and the obtained stress-strain curves are shown in Figure $3 b$. Consistent with previous studies, ${ }^{[31]}$ the remarkable lattice orientation dependence for the strength is observed (see Figure 3c). As the mechanical properties of graphene are usually closely related to the lattice orientation, the fracture behavior as a function of the lattice orientation should be investigated. To this end, graphene sheets with central cracks of different sizes are stretched along given lattice orientations (note that the crack line is always perpendicular to the loading direction), and the corresponding evolution of the critical fracture stress with respect to the crack length is shown in Figure $3 \mathrm{~d}$. It can be seen that with graphene samples involving central cracks the critical fracture stress is much less than the one of its perfect form as anticipated, and its evolution with respect to the crack length is well fitted by $\sigma_{f}=\mathrm{Ca}^{-\alpha}$, where the singular exponent $\alpha$ as a function of the lattice orientation is shown in Figure 3e. Different from the monotonous lattice orientation dependence of strength, the maximum of the singular exponent $\alpha$ is obtained by stretching along the lattice orientation with $\varphi=15^{\circ}$. a

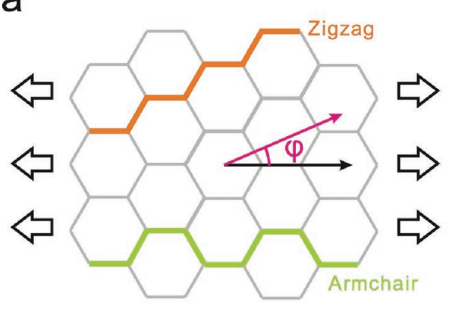

b
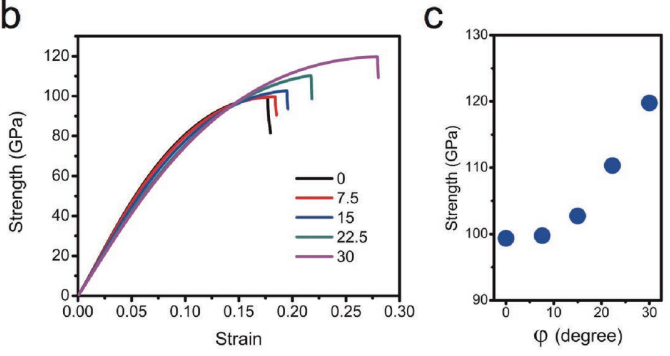

d

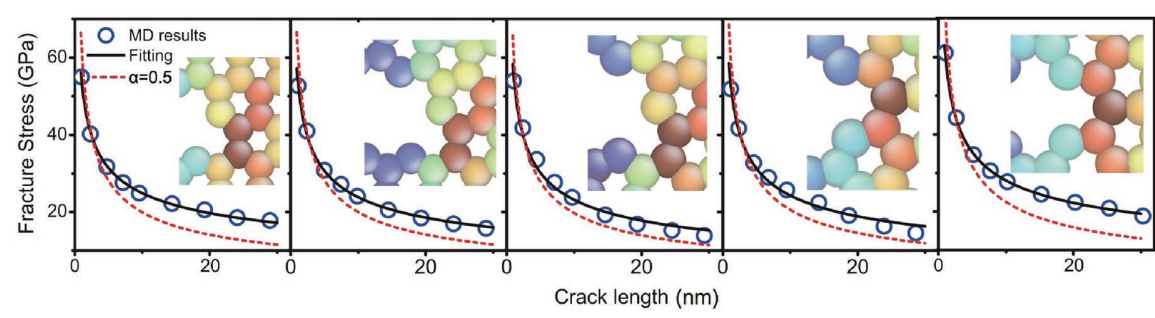

e
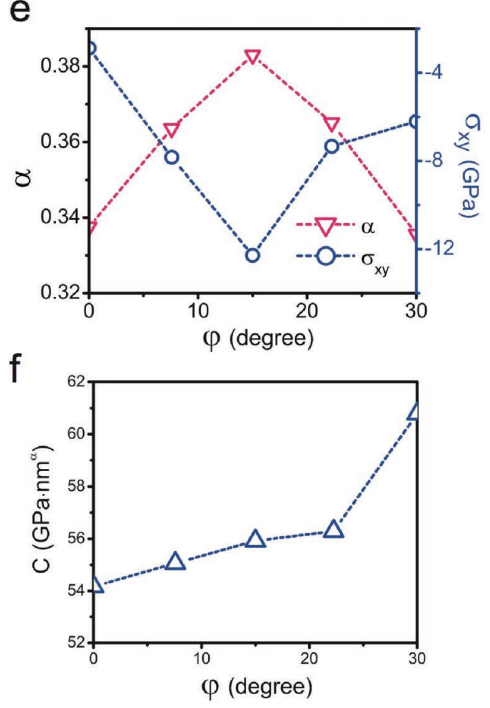

Figure 3. Lattice orientation dependence of mechanical properties (the strength and fracture behavior) in graphene. a) The schematic representation of the lattice orientation in graphene, where the angle $\varphi$ is used to define the lattice orientation. For example, the shown loading direction is along the armchair direction, and the corresponding angle $\varphi=0^{\circ}$. b) The stress-strain curves for graphene subject to tension for $\varphi=0^{\circ}, 7.6^{\circ}, 15^{\circ}, 22.3^{\circ}$, $30^{\circ}$. c) The evolution of strength with respect to $\varphi$. d) The critical fracture stress versus the crack length. Note that the loading direction changes from armchair to zigzag, and $\varphi=0^{\circ}, 7.6^{\circ}, 15^{\circ}, 22.3^{\circ}, 30^{\circ}$ from left to right. Note that MD results (blue circles) are well fitted by $\sigma_{f}=C a^{-\alpha}(\mathrm{black}$ solid line) and the insets show the typical atomic structure at the crack tip before the onset of the fracture. For comparison, the fitting with $\alpha=0.5$ (according to Griffith criterion) is provided (the red dash line) in (d). e) The corresponding singular exponent $\alpha$ and the stress $\sigma_{x y}$ of the bond at the crack tip versus $\varphi$. f) The coefficient C versus $\varphi$. 
One issue to be aware of is that the maximum of the singular exponent $\alpha(0.384)$ is very close to the singular exponent 0.3843 for the $120^{\circ}$ notch (predicted by Equation (1)), which implies in that case not only the bond angles at the crack tip but also the bond angles nearby are combined to determine the fracture behavior. It is believed that the level of the lattice distortion, i.e., relative motion of atoms along the horizontal axis, at the crack tip determines the number of the involved bond angles and thus the magnitude of the singular exponent $\alpha$. With $1.5 \%$ tensile strain applied to samples containing central cracks about $20 \mathrm{~nm}$ in length, the stress $\sigma_{x y}$ of the atomic bond at the crack tip as the driving force of the lattice distortion is used to reflect the level of the lattice distortion, and its lattice orientation dependence is shown in Figure 3e. Note that the stress $\sigma_{x y}$ of a bond is obtained by averaging the stresses $\sigma_{x y}$ at the two atoms. As the singular exponent $\alpha$ is found to have positive correlation with the magnitude of the stress $\sigma_{x y}$, our speculation is validated and could be used to explain the lattice orientation dependence of the singular exponent $\alpha$ as follows.

In the case of stretching along the zigzag or armchair direction, the lattice structure near the crack tip (see the insets in Figure 3d) is nearly symmetric respect to the horizontal axis, and thus the lattice distortion at the crack tip is quite small; however, gradually deviating from these two special loading directions, this distortion becomes more and more serious, and thus much more bond angles near the crack tip are involved in the fracture process region. Since the bond angles near the crack tip are close to $120^{\circ}$, the singular exponent $\alpha$ increases and approaches to 0.3843 with the increasing deviation between the loading direction and special lattice orientations, i.e., the armchair and zigzag direction.
Besides, the coefficient $\mathrm{C}$ obtained by fitting as a function of the lattice orientation is shown in Figure $3 \mathrm{f}$, which could not give much information considering the different dimension of the coefficient $\mathrm{C}$ for different lattice orientations. Overall, the lattice orientation dependent fracture behavior further confirms the intrinsic notch effect and also the breakdown of the Griffith criterion in graphene.

\subsection{Effect of External Notch}

As the crack blunting in practice is inevitable, where a sharp crack tip is substituted by a blunting notch tip (see Figure 4a), several authors have studied the accompanied notch effect in graphene. ${ }^{[24,39,40]}$ One of these works ${ }^{[24]}$ focused on the influence of the notch radius to the fracture toughness and reported that the increasing notch radius could improve the fracture toughness. Note that since this notch effect could be artificial, it is hereinafter called the external notch effect, and the relevant issue here is whether the external notch effect could mask the intrinsic one. To figure it out, samples containing central cracks blunted with $2.5 \mathrm{~nm}$ notch radii are prepared (see Figure 4a), then the tensile test is carried out to investigate the fracture behavior. With a $20 \mathrm{~nm}$ crack along the zigzag direction in the sample, the atomic structure near the external notch at $3 \%$ tensile strain is shown in Figure $4 \mathrm{~b}$ (the vicinity of the notch tip is magnified in Figure 4c), and the corresponding stress distribution ahead of the notch tip is given in Figure 4d. Although comparing with the sharp crack tip, the stress concentration near the external notch decreases, the fracture still initiates from the breakage of one carbon-carbon bond (see Figure S3, Supporting information), a

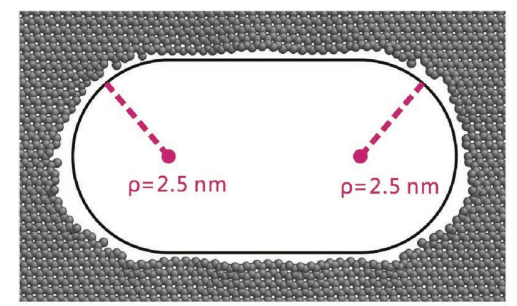

b

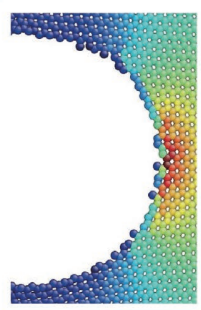

C

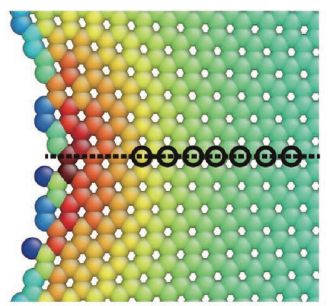

d

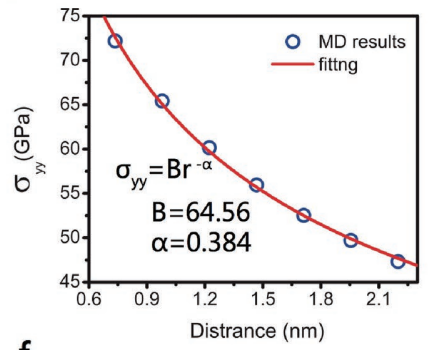

$f$

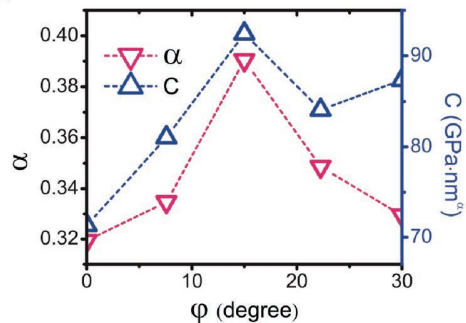

$\varphi$ (degree)

e

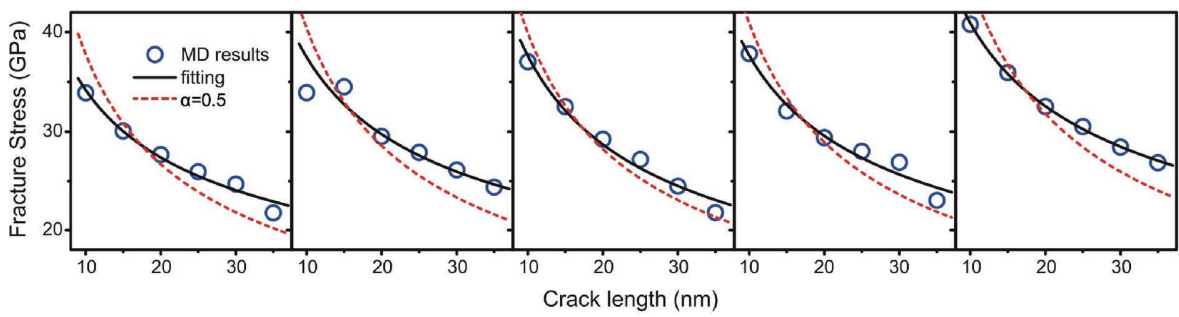

Figure 4. Fracture behavior in graphene with external notches. a) The representative graphene sample where a $10 \mathrm{~nm}$ central crack is blunted with $2.5 \mathrm{~nm}$ notch radii. b) The atomic structure near the notch tip in the sample containing a central crack of $20 \mathrm{~nm}$ subject to $3 \%$ tensile strain. The color reflects the distribution of the virial stress $\sigma_{y y}$. c) Zoom in the vicinity of the notch tip. d) The stress distribution along the horizontal direction and the corresponding atoms are marked with black circles in (c). e) The critical fracture stress versus the crack length, note that the loading direction changes from armchair to zigzag. Specifically, $\varphi=0^{\circ}, 7.6^{\circ}, 15^{\circ}, 22.3^{\circ}, 30^{\circ}$ from left to right. Note that MD results (blue circles) are well fitted by $\sigma_{f}=\mathrm{Ca}^{-\alpha}$ (black solid line), and the evolution of the singular exponent $\alpha$ and coefficient $\mathrm{C}$ with respect to $\varphi$ is given in (f). For comparison, the fitting with $\alpha=0.5$ (according to Griffith criterion) is provided (the red dash line) in (e). 
and thus the intrinsic notch effect could be dominant. Tensile tests along different lattice orientations are performed, and the evolution of the critical fracture stress with respect to the crack length is given in Figure 4e, which is well fitted by $\sigma_{f}=C a^{-\alpha}$. The corresponding singular exponent $\alpha$ as a function of the lattice orientation (changes from 0.32 to 0.39 ) can be found in Figure 4f, which is similar to the case in the last section (see Figure 3e). Therefore, it concludes that the external notch effect would not mask the intrinsic one in graphene.

\subsection{Experimental Evidence}

Recently, a direct and quantitative approach to test the fracture properties of graphene is reported, ${ }^{[24]}$ leading to the first direct experimental measurement of fracture toughness in graphene. ${ }^{[41]}$ To the best of our knowledge, it might be the unique one until now. As demonstrated by our theoretical analysis and MD simulations, the intrinsic notch effect is robust and thus should be observed in the experiment. Here we simply assume that the geometry setting in the experiments does not change the form of the fracture criterion in the stress intensity factor theory, except an additional modifying factor $f$. To be specific, $\sigma_{f} \sim f(H / a, W / a \ldots) K_{I} a^{-\alpha} \sim C a^{-\alpha}$, where $H$ and $W$ are the length and width of the sample in experiments, respectively. As shown in Figure 5, the experimental result is well fitted by the above equation with $C=48.8 \mathrm{GPa} \cdot n m^{\alpha}$ and $\alpha=0.39$, and it is encouraging to find out that the value of the singular exponent $\alpha$ is within the range obtained by our MD simulations. Specially, it is in good agreement with the MD results for the loading direction along the lattice orientation with $\varphi=15^{\circ}$ (see Figure $3 \mathrm{e}, \mathrm{f}$ ), where $C=56 \mathrm{GPa} \cdot n m^{\alpha}$ and $\alpha=0.384$. As anticipated, the intrinsic notch effect is roust and obvious in the experiment rather than the external one. For comparison, the fitting with $\alpha=0.5$ (according to Griffith criterion) is provided in Figure 5, which could overestimate the fracture stress for a short crack and underestimate it for a long crack.

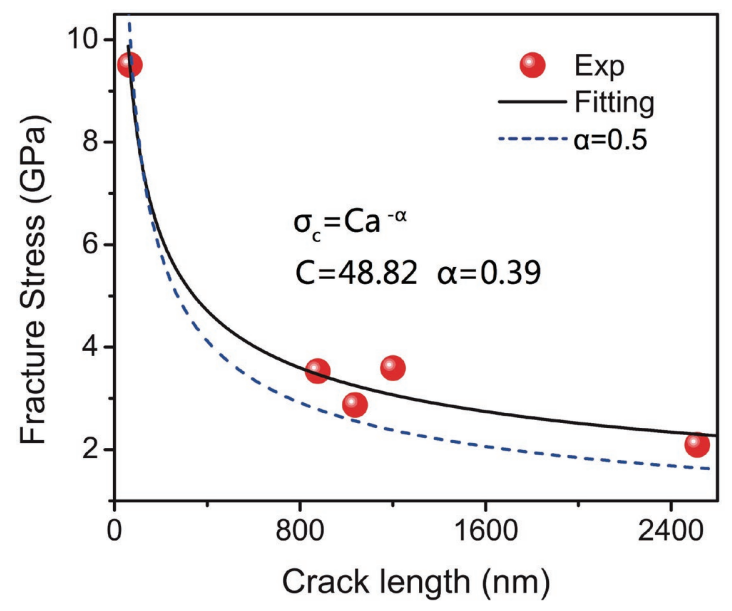

Figure 5. The experimental evidence. The critical fracture stress versus the crack length, where the red dots are experimental data and are well fitted by $\sigma_{f}=\mathrm{Ca}^{-\alpha}$ (the black solid line). For comparison, the fitting with $\alpha=0.5$ (according to Griffith criterion) is provided (the blue dash line).

\section{Discussion}

In the Griffith theory, only Young's modulus $E$ and surface energy $\Gamma$ parameters are involved to capture the fracture behavior. For a central crack of length $a$ in an infinite body, the critical fracture stress $\sigma_{f} \sim \sqrt{\Gamma E / a}$ could be derived according to the Griffith criterion. ${ }^{[37]}$ As the dimensional analysis suggests, this criterion should remain if no more length scales are involved. Unfortunately, with the decrease of the thickness, the more pronounced lattice structure introduces the bond length as well as the bond pattern into this question, leading to the breakdown of the Griffith criterion.

As a manifestation of the breakdown of the Griffith criterion, the intrinsic notch effect in graphene is first recognized and validated through the comparisons between theoretical calculations and experimental data. Since the intrinsic notch effect decreases the stress singularity at the crack tip, it should increase the fracture toughness in graphene. Note that not only for graphene but also for those 2D materials lack of plastic mechanisms, the intrinsic notch effect could work, and as a result Griffith criterion breaks down. On the opposite, with the size of the plastic region larger than nano scale but much smaller than the length of the crack, the intrinsic notch effect could be buried, and in this case Griffith criterion should recover. Moreover, the angle of the intrinsic notch determines the derivation from Griffith criterion, and if we focus on the fracture of Mode I, the intrinsic notch effect, which could be negligible with its angle less than $60^{\circ}$, gradually becomes remarkable as the angle increased (see the black line in Figure $1 \mathrm{c}$ and note that the singular exponent $\alpha=1-\lambda$ is very close to 0.5 when the intrinsic notch angle is less than $60^{\circ}$ ). In this sense, the obvious intrinsic notch effect in graphene benefits from its $120^{\circ}$ bond angles. Due to lack of plastic deformation ability for the vast majority of 2D materials, their lattice structures remain until the extension of the crack, which provide a prerequisite for realizing the intrinsic notch effect. Therefore, not only in graphene, it is believed that in 2D materials this intrinsic notch effect is ubiquitous underscoring the critical need for exploring the fracture behavior of 2D materials both theoretically and experimentally.

One thing should be noted is that the intrinsic notch effect in this article is essentially different from the concept of lattice trapping proposed by Thomson. ${ }^{[42,43]}$ First, they have different origins. The intrinsic notch effect originates from the bond angles near the crack tip, while lattice trapping is derived from the oscillating surface energy due to the discrete atomic structure. Second, their consequences are distinct. In graphene, the intrinsic notch effect could lead to a different fracture criterion $\sigma_{f} \sim a^{-\mathrm{a}}$ deviating from the Griffith criterion $\sigma_{f} \sim a^{-1 / 2}$. However, lattice trapping results in the enhancement of the fracture toughness, but Griffith criterion could remain.

Theoretically, the intrinsic notch effect in fracture of graphene is believed to mainly stem from two factors. The first one is the honeycomb lattice structure, since it provides the considerable large bond angle $\left(120^{\circ}\right)$, the intrinsic notch effect becomes obvious and robust. The second one is lack of plasticity in graphene, which makes the intrinsic notch remain 
until the extension of the crack. Since both these two factors could be reproduced in metamaterials with an accurate design, the intrinsic notch effect learnt from graphene could be utilized in metamaterials, which is the artificial micro- and nanoarchitected materials assembled from one or multiple kinds of materials in repeating patterns and could be even engineered to have a property that is not found in nature. Recently, lightweight mechanical metamaterials have made rapid progress and gained academic attention. ${ }^{[44-47]}$ As current discussions are focused on the specific strength (the ratio of strength to density) of these metamaterials, their fracture toughness is believed to be the next target mechanical index, which is much more engineering relevant. It suggests that the design with large "bond angles" in these artificial lattice could be used to reduce the stress concentration near the flaw, and our results are thus supposed to provide a feasible strategy toward tuning the fracture toughness of metamaterials.

\section{Conclusion}

In conclusion, based on MD simulations we first recognize that the carbon-carbon bond angle in graphene serves as an intrinsic notch with infinite acuity in graphene, the corresponding notch effect, called the intrinsic notch effect, changes the stress singularity near the crack tip as well as the fracture behavior leading to the breakdown of the Griffith criterion. Further, the lattice orientation dependence of the fracture behavior and the influence of the external notch effect are investigated witnessing the existence and robustness of the intrinsic notch effect. Particularly, the intrinsic notch effect could be used to well explain the recent experimental data, which is considered as the first direct experimental study of the fracture behavior in graphene. Essentially, if the stress field is nonuniform (could be caused by chemical modification, local loading, defects, etc.) in an extremely brittle material, where the characteristic length scale of the plastic region is close to the atomic length scale, the intrinsic notch effect could work. Its fundamental significance is profound and far reaching. For example, the strength of polycrystalline $2 \mathrm{D}$ materials, nanoindention tests in $2 \mathrm{D}$ materials, and even the fracture toughness of metamaterials could be closely related to the intrinsic notch effect proposed in this article. We believe that it paves a way to a more comprehensive and deep understanding of the unique mechanical properties in nano as well as nanostructured materials.

\section{Experimental Section}

MD simulations were performed using in large-scale atomic/ molecular massively parallel simulator ${ }^{[48]}$ based on the adaptive intermolecular reactive empirical bond-order (AIREBO) potential, ${ }^{[49]}$ with the interaction cutoff parameter set to $2 \AA .{ }^{[50,51]}$ All graphene samples (about $95 \mathrm{~nm} \times 85 \mathrm{~nm}$ ) used in the strength and toughness simulations were prepared by running NPT (where the number of particles $N$, pressure $P$, and temperature $T$ of the system are specified)dynamics at $1 \mathrm{~K}$ for $100 \mathrm{ps}$ to allow the relaxation of the simulation box dimensions to attain the zero stress state, and then the uniaxial tensile test was performed with a strain rate of $0.001 \mathrm{ps}^{-1}$. Note that a time step of $1 \mathrm{fs}$ and periodic boundary condition were used in all of the simulations. Besides, NVT (where the number of particles N, volume V, and temperature $\mathrm{T}$ of the system are specified) ensemble was applied in the tensile test, where temperature was maintained at $1 \mathrm{~K}$.

Note that since the atomic environment near a crack tip deviated strongly from the equilibrium bonding situation, empirical potentials might fail to correctly describe the fracture behavior of specific materials. ${ }^{[43]}$ Therefore, the results from the AIREBO potential and first principle calculations for the localized structure near the crack tip were directly compared to confirm that the AIREBO potential could correctly describe the energy landscape of the atom at the crack tip (the details could be found in the Supporting Information). As it decided the bond breaking at the crack tip and thus the fracture strength of graphene, the validity of the $M D$ results for brittle fracture in graphene could be demonstrated.

\section{Supporting Information}

Supporting Information is available from the Wiley Online Library or from the author.

\section{Acknowledgements}

The research was supported by the National Natural Science Foundation of China (Grant Nos. 11602272, 11602270, 11021262, 11172303, 11132011, and 11532013), the National Basic Research Program of China ("973" Project) (Grant No. 2012CB937500), and the Strategic Priority Research Program of the Chinese Academy of Sciences (Grant No. XDB22040503).

\section{Conflict of Interest}

The authors declare no conflict of interest.

[1] A. C. Neto, F. Guinea, N. M. Peres, K. S. Novoselov, A. K. Geim, Rev. Mod. Phys. 2009, 81, 109.

[2] K. Novoselov, A. K. Geim, S. Morozov, D. Jiang, M. Katsnelson, I. Grigorieva, S. Dubonos, A. Firsov, Nature 2005, 438, 197.

[3] A. A. Balandin, S. Ghosh, W. Bao, I. Calizo, D. Teweldebrhan, F. Miao, C. N. Lau, Nano Lett. 2008, 8, 902.

[4] F. Bonaccorso, Z. Sun, T. Hasan, A. Ferrari, Nat. Photonics 2010, 4, 611.

[5] C. Lee, X. Wei, J. W. Kysar, J. Hone, Science 2008, 321, 385.

[6] G.-H. Lee, R. C. Cooper, S. J. An, S. Lee, A. van der Zande, N. Petrone, A. G. Hammerberg, C. Lee, B. Crawford, W. Oliver, Science 2013, 340, 1073.

[7] S. Bae, H. Kim, Y. Lee, X. Xu, J.-S. Park, Y. Zheng, J. Balakrishnan, T. Lei, H. R. Kim, Y. I. Song, Nat. Nanotechnol. 2010, 5, 574.

[8] G. Eda, G. Fanchini, M. Chhowalla, Nat. Nanotechnol. 2008, 3, 270.

[9] K. S. Kim, Y. Zhao, H. Jang, S. Y. Lee, J. M. Kim, J. H. Ahn, P. Kim, J. Y. Choi, B. H. Hong, Nature 2009, 457, 706. 
[10] J. S. Bunch, A. M. Van Der Zande, S. S. Verbridge, I. W. Frank, D. M. Tanenbaum, J. M. Parpia, H. G. Craighead, P. L. McEuen, Science 2007, 315, 490.

[11] K. S. Novoselov, V. Fal, L. Colombo, P. Gellert, M. Schwab, K. Kim, Nature 2012, 490, 192.

[12] R. Roldán, A. Castellanos-Gomez, E. Cappelluti, F. Guinea, J. Phys.: Condens. Matter 2015, 27, 313201.

[13] L. Tapasztó, T. Dumitrică, S. J. Kim, P. Nemes-Incze, C. Hwang, L. P. Biró, Nat. Phys. 2012, 8, 739.

[14] W. Bao, F. Miao, Z. Chen, H. Zhang, W. Jang, C. Dames, C. N. Lau, Nat. Nanotechnol. 2009, 4, 562.

[15] K.-K. Bai, Y. Zhou, H. Zheng, L. Meng, H. Peng, Z. Liu, J.-C. Nie, L. He, Phys. Rev. Lett. 2014, 113, 086102.

[16] C. Wang, Y. Liu, L. Lan, H. Tan, Nanoscale 2013, 5, 4454.

[17] Z. Wang, M. Devel, Phys. Rev. B 2011, 83, 125422.

[18] F. Liu, T.-C. Wang, Carbon 2016, 96, 1175.

[19] A. Omeltchenko, J. Yu, R. K. Kalia, P. Vashishta, Phys. Rev. Lett. 1997, 78, 2148.

[20] Y. Jin, F. Yuan, J. Nanosci. Nanotechnol. 2005, 5, 2099.

[21] R. Khare, S. L. Mielke, J. T. Paci, S. Zhang, R. Ballarini, G. C. Schatz, T. Belytschko, Phys. Rev. B 2007, 75, 075412.

[22] S. S. Terdalkar, S. Huang, H. Yuan, J. J. Rencis, T. Zhu, S. Zhang, Chem. Phys. Lett. 2010, 494, 218.

[23] M. Xu, A. Tabarraei, J. T. Paci, J. Oswald, T. Belytschko, Int. J. fract. 2012, 173, 163

[24] P. Zhang, L. Ma, F. Fan, Z. Zeng, C. Peng, P. E. Loya, Z. Liu, Y. Gong, J. Zhang, X. Zhang, Nat. Commun. 2014, 5, 3782

[25] Z. Zhang, X. Wang, J. D. Lee, J. Appl. Phys. 2014, 115, 114314.

[26] M. Dewapriya, R. Rajapakse, J. Appl. Mech. 2014, 81, 081010.

[27] T. Zhang, X. Li, S. Kadkhodaei, H. Gao, Nano Lett. 2012, 12, 4605.

[28] G. Jung, Z. Qin, M. J. Buehler, Extreme Mech. Lett. 2015, 2, 52.

[29] G. Lopez-Polin, J. Gomez-Herrero, C. Gomez-Navarro, Nano Lett. 2015, 15, 2050.

[30] Y. Hwangbo, C. K. Lee, S. M. Kim, J. H. Kim, K. S. Kim, B. Jang, H. J. Lee, S. K. Lee, S. S. Kim, J. H. Ahn, S. M. Lee, Sci. Rep. 2014 4, 4439.
[31] H. Yin, H. J. Qi, F. Fan, T. Zhu, B. Wang, Y. Wei, Nano Lett. 2015, 15, 1918.

[32] N. M. Pugnot, R. S. Ruoff, Philos. Mag. 2004, 84, 2829.

[33] M. Wang, C. Yan, L. Ma, N. Hu, M. Chen, Comput. Mater. Sci. 2012, $54,236$.

[34] H. Zhao, N. Aluru, J. Appl. Phys. 2010, 108, 064321.

[35] U. A. Joshi, S. C. Sharma, S. Harsha, Physica E 2011, 43, 1453.

[36] G. Pluvinage,Fracture and Fatigue Emanating from Stress Concentrators, Kluwer Publisher, Dordrecht, Netherlands 2003.

[37] A. A. Griffith, Philos. Trans. R. Soc., A 1921, 221, 163.

[38] F. Liu, P. Ming, J. Li, Phys. Rev. B 2007, 76, 064120.

[39] S. Kumar, M. Haque, H. Gao, Appl. Phys. Lett. 2009, 94, 253104.

[40] Z. Sha, Q. Pei, Z. Liu, V. Shenoy, Y. Zhang, Carbon 2014, 72, 200.

[41] T. Zhang, X. Li, H. Gao, Int. J. Fract. 2015, 196, 1.

[42] R. Thomson, C. Hsieh, V. Rana, J. Appl. Phys. 1971, 42, 3154.

[43] E. Bitzek, J. R. Kermode, P. Gumbsch, Int. J. Fract. 2015, 191, 13.

[44] T. A. Schaedler, A. J. Jacobsen, A. Torrents, A. E. Sorensen, J. Lian, J. R. Greer, L. Valdevit, W. B. Carter, Science 2011, 334, 962.

[45] X. Zheng, H. Lee, T. H. Weisgraber, M. Shusteff, J. DeOtte, E. B. Duoss, J. D. Kuntz, M. M. Biener, Q. Ge, J. A. Jackson, Science 2014, 344, 1373.

[46] L. R. Meza, S. Das, J. R. Greer, Science 2014, 345, 1322.

[47] X. Li, H. Gao, Nat. Mater. 2016, 15, 373.

[48] S. Plimpton, J. Comput. Phys. 1995, 117, 1.

[49] S. J. Stuart, A. B. Tutein, J. A. Harrison, J. Chem. Phys. 2000, 112, 6472.

[50] O. Shenderova, D. Brenner, A. Omeltchenko, X. Su, L. Yang, Phys. Rev. B 2000, 61, 3877.

[51] Z. Xu, J. Comput. Theor. Nanosci. 2009, 6, 625.

Received: January 3, 2017

Revised: March 3, 2017

Published online: May 16, 2017 